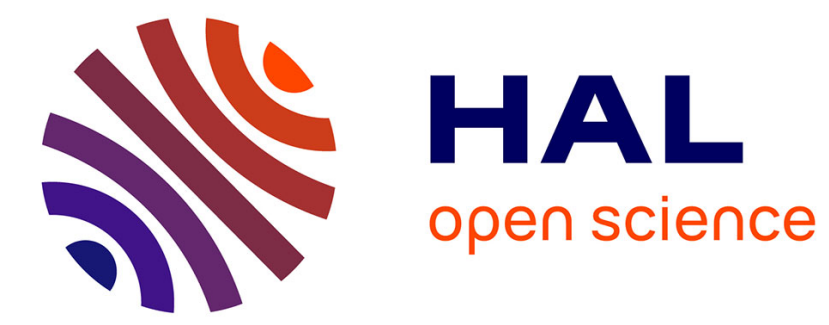

\title{
Is there seismic attenuation in the mantle?
}

Y Ricard, S Durand, J.-P Montagner, F Chambat

\section{To cite this version:}

Y Ricard, S Durand, J.-P Montagner, F Chambat. Is there seismic attenuation in the mantle?. Earth and Planetary Science Letters, 2014, 388, pp.257 - 264. 10.1016/j.epsl.2013.12.008 . hal-02944834

\section{HAL Id: hal-02944834 \\ https://hal.science/hal-02944834}

Submitted on 7 Dec 2020

HAL is a multi-disciplinary open access archive for the deposit and dissemination of scientific research documents, whether they are published or not. The documents may come from teaching and research institutions in France or abroad, or from public or private research centers.
L'archive ouverte pluridisciplinaire HAL, est destinée au dépôt et à la diffusion de documents scientifiques de niveau recherche, publiés ou non, émanant des établissements d'enseignement et de recherche français ou étrangers, des laboratoires publics ou privés. 


\title{
Is there seismic attenuation in the mantle?
}

\author{
Y. Ricard ${ }^{\mathrm{a}, *}$, S. Durand ${ }^{\mathrm{a}}$, J.-P. Montagner ${ }^{\mathrm{b}}$, F. Chambat ${ }^{\mathrm{a}}$ \\ a Laboratoire de Géologie de Lyon - Terre Planète Environnement, CNRS UMR5570, École Normale Supérieure de Lyon, Université de Lyon, \\ Université Claude Bernard Lyon 1, 46 Allée d'Italie, 69364 Lyon Cedex 07, France \\ b Institut de Physique du Globe de Paris, Université Paris-Diderot, 1 rue Jussieu, 75238 Paris Cedex 05, France
}

\section{A R T I C L E I N F O}

\section{Article history:}

Received 23 August 2013

Received in revised form 3 December 2013

Accepted 6 December 2013

Available online 31 December 2013

Editor: P. Shearer

\section{Keywords:}

apparent attenuation

$Q$

scattering

random media

\begin{abstract}
A B S T R A C T
The small scale heterogeneity of the mantle is mostly due to the mixing of petrological heterogeneities by a smooth but chaotic convection and should consist in a laminated structure (marble cake) with a power spectrum $S(k)$ varying as $1 / k$, where $k$ is the wavenumber of the anomalies. This distribution of heterogeneities during convective stirring with negligible diffusion, called Batchelor regime is documented by fluid dynamic experiments and corresponds to what can be inferred from geochemistry and seismic tomography. This laminated structure imposes density, seismic velocity and potentially, anisotropic heterogeneities with similar $1 / k$ spectra. A seismic wave of wavenumber $k_{0}$ crossing such a medium is partly reflected by the heterogeneities and we show that the scattered energy is proportional to $k_{0} S\left(2 k_{0}\right)$. The reduction of energy for the propagating wave appears therefore equivalent to a quality factor $1 / Q \propto k_{0} S\left(2 k_{0}\right)$. With the specific $1 / k$ spectrum of the mantle, the resulting apparent attenuation should therefore be frequency independent. We show that the total contribution of 6-9\% RMS density, velocity and anisotropy would explain the observed $\mathrm{S}$ and $\mathrm{P}$ attenuation of the mantle. Although these values are large, they are not unreasonable and we discuss how they depend on the range of frequencies over which the attenuation is explained. If such a level of heterogeneity were present, most of the attenuation of the Earth would be due to small scale scattering by laminations, not by intrinsic dissipation. Intrinsic dissipation must certainly exist but might correspond to a larger, yet unobserved $Q$. This provocative result would explain the very weak frequency dependence of the attenuation, and the fact that bulk attenuation seems negligible, two observations that have been difficult to explain for 50 years.
\end{abstract}

(c) 2013 Elsevier B.V. All rights reserved.

\section{Introduction}

After reviewing laboratory and seismological observations, Knopoff (1964) concluded that the seismic quality factor $Q$ (or attenuation $Q^{-1}$ ) depended only weakly on the frequency $\omega$. This observation was not easily compatible with the theoretical models developed for the anelastic behavior. Indeed, these models predicted a frequency dependent behavior with a maximum of absorption centered on a frequency related to the relaxation time of a given mechanism. Later, Jackson and Anderson (1970) and Liu et al. (1976) proposed to explain this quasi frequency-independent behavior by the superposition of standard linear solids whose relaxation times covered the observed absorption band.

In the last 30 years, seismological studies have however identified some frequency dependence of the attenuation. From normal modes and surface waves (say in the range $0.001-0.05 \mathrm{~Hz}$ ), a weak dependence of the attenuation has been proposed with $Q^{-1} \propto \omega^{\alpha}$ and $\alpha \approx-0.2$ (e.g., Lekic et al., 2009). An expo-

\footnotetext{
* Corresponding author. Tel.: +33 (0)4 72448413.

E-mail address: ricard@ens-lyon.fr (Y. Ricard).
}

nent in the same range $(-0.4 \leqslant \alpha \leqslant 0)$ has been found using body waves up to $\approx 1 \mathrm{~Hz}$ (e.g., Choy G. L. and Cormier, 1986; Shito et al., 2004). Somewhere above $1 \mathrm{~Hz}$ there is strong evidence of a corner past which the exponent becomes closer to $\alpha \approx-1$ (Choy G. L. and Cormier, 1986; Cormier, 2011). On the low frequency side, below $0.001 \mathrm{~Hz}$, the attenuation is likely increasing moderately with $\alpha \approx 0.4$ (Lekic et al., 2009). Within a large frequency domain, $10^{-4}-1 \mathrm{~Hz}$, the attenuation varies therefore by less than an order of magnitude.

A modest frequency dependence of the attenuation $(\alpha \approx-0.27)$ has also been observed in laboratory experiments with polycrystalline aggregates of olivine (Jackson et al., 2002; Faul and Jackson, 2005). The similarity of exponents found in laboratory experiments and in seismological observations suggests that similar dissipation mechanisms might be present in the two situations. The laboratory experiments are however performed under conditions that are not identical to the seismologic situation (viscoelastic torsion rather than seismic propagation, smaller material grain sizes, larger strain rates, much smaller scale, ...). Several possible micromechanisms of attenuation have been suggested (see Jackson, 2007 for a review); it is only by a specific combinations of them, distributed 
over a large attenuation band that the seismic observations can be accounted for.

The attenuation in the mantle seems to be mostly due to shear attenuation $Q_{\mu}^{-1}$ ( $\mu$ is rigidity) while bulk attenuation $Q_{\kappa}^{-1}(\kappa$ is incompressibility) is much smaller (e.g., Romanowicz and Mitchell, 2006). This behavior is surprising for the following reason. Submitting an elastic medium to extension results in a perpendicular deformation generally in compression and controlled by a positive Poisson's ratio $v=(3 \kappa-2 \mu) /(6 \kappa+2 \mu)$. Therefore, for most materials, $3 \kappa>2 \mu$ (this is not a thermodynamic rule but simply an empirical observation; some rare materials called auxetic have a negative Poisson's ratio). For a dissipative medium submitted to a slow stretching, one would also expect the perpendicular velocity to be similarly in compression. For a linear solid, the correspondence principle relates the velocities to the deformations by replacing the real elastic parameters $\kappa$ and $\mu$ by their imaginary counterparts $\kappa Q_{\kappa}^{-1}$ and $\mu Q_{\mu}^{-1}$. Therefore one would expect $3 \kappa Q_{\kappa}^{-1}>2 \mu Q_{\mu}^{-1}$ or $Q_{\kappa}^{-1}>(2 \mu) /(3 \kappa) Q_{\mu}^{-1}$ (Morozov, 2013); a $Q_{\kappa}^{-1}$ of order $0.2 Q_{\mu}^{-1}$ or larger would be expected rather than the surprising $Q_{\kappa}^{-1} \approx 0$.

The attenuation measured by seismologists is in fact a combination of various mechanisms. Some are really dissipative (i.e., they convert the elastic energy into heat), some are due to various non-dissipative effects (i.e., the coherent elastic energy is refracted, scattered into incoherent signals, defocused, ...). In the latter case, the coherent elastic energy is lost for a direct observation but remains distributed in the Earth (before being eventually dissipated in the fluid and solid envelopes of the Earth). This "elastic" attenuation is hard to quantify and makes the measurements of intrinsic attenuation difficult for body waves and surface waves (see review by Romanowicz and Mitchell, 2006; Shearer and Earle, 2008). Attenuation can also be derived with normal modes from the width of spectral peaks (Dahlen, 1982). Mode coupling by heterogeneities broadens the spectral peaks and again, separating this effect from intrinsic attenuation is complex. A similar difficulty for separating intrinsic and extrinsic phenomena exists also for anisotropy (Wang et al., 2013; Fichtner et al., 2013).

In this paper we will estimate the elastic attenuation that can be due to the heterogeneities in density, velocity or anisotropy of the mantle. We show that the specific spectrum of the heterogeneities in the mantle implies, as it is observed, that the $\mathrm{P}$ and $\mathrm{S}$ elastic attenuations should be frequency independent and that the $\mathrm{P}$ attenuation should be likely smaller than the $\mathrm{S}$ attenuation. These attenuations interpreted in terms of $Q_{\kappa}, Q_{\mu}$ agree with $Q_{\kappa} \gg Q_{\mu}$ without implying a surprising auxetic rheology for the mantle. In order to reach the typical observations of attenuation in the mantle, heterogeneities of 6-9\% in density, velocity and anisotropy are needed. These values are very large but might not be unreasonable. For a lower level of heterogeneities, intrinsic attenuation would dominate a frequency independent elastic attenuation.

\section{Heterogeneities in the mantle}

The smooth large scale heterogeneities of the mantle are likely due to lateral temperature variations related to thermal convection. However at small length scale there are more certainly related to petrological/compositional anomalies. As thermal diffusivity is much larger than chemical diffusivity, the gradients of composition remain indeed much sharper than those of temperature. Compositional heterogeneities, like thermal ones, induce density, velocity but also anisotropy variations. The origin of compositional variations in the mantle could be due to a primordial layering of the mantle and more obviously to the continuous injection of oceanic lithosphere in the mantle (Coltice and Ricard, 1999). The difference in density or velocity between oceanic crust and depleted harzburgite reaches $10 \%$ and although these two components undergo various phase changes when the lithospheric slab sinks in the mantle, contrasts of several \% should remain throughout the mantle (Ricard et al., 2005). The presence of localized melt bands (in the upper mantle at least), with $5 \%$ or more impedance contrasts has also been observed (Kawakatsu et al., 2011; Tauzin et al., 2010).

The mixing of heterogeneities in chaotic convecting fluids has been studied for a long time. In situations appropriate for the Earth, when heterogeneities are continuously injected on a length scale smaller than that of a flow which is smooth but chaotic, the heterogeneity power spectrum should vary like $1 / k$ where $k$ is the wavenumber of the heterogeneity $(k=2 \pi / \lambda$ where $\lambda$ is the wavelength). This result was obtained by Batchelor (1959) and is sometimes called "Batchelor rule". These steady state results have been extended and confirmed for initial value problems (Antonsen and Ott, 1991). The basic physics that leads to this result is rather simple to explain (Olson et al., 1984). The homogeneization in the mantle does not occur by diffusing away the heterogeneities but rather by stirring them. Advected by the flow, a heterogeneity of wavelength $\lambda$, is stretched and folded (the so-call "baker" transformation, where the pastry maker kneads the dough is prototypical of a mixing process). A heterogeneity is therefore transformed into a thin sheet multiply folded. This process continuously reduces the wavelength of the heterogeneities and the energy cascades down the power spectrum toward the large wavenumbers. The injection replenishes the low wavenumber spectrum and in steady state, a $1 / k$ spectrum results.

Several authors have tried to infer the power spectrum of the mantle from geochemical or seismic observations. From isotopic $\mathrm{Sr}$ variations of ridge basalts, Gurnis (1986) suggested that the power spectrum of the mantle may be rather flat ("white") which would imply a drastic heterogeneity at short wavelength. Using a similar approach but with orders of magnitude more data and several isotopic ratios, Agranier et al. (2005) observed a clear $1 / k$ spectrum along much of the Atlantic ridge.

Long wavelength tomography probably maps thermal heterogeneity that may decrease faster than a $1 / k$ spectrum (Montagner, 1994). However this decrease is partially due to the regularization of the inversion (Ricard et al., 1996) and a spectrum closer to $1 / k$ is also obtained by patching together global and regional tomographies (Chevrot et al., 1998). A more precise estimate of the short wavelength content of the mantle comes from fitting the amplitude of PKP precursors in the mantle. Following the pioneering works of Cormier (1995) and Hedlin et al. (1997), a study by Margerin and Nolet (2003) found small RMS P velocity (0.1-0.2\%) in the deep Earth. This low level of short wavelength $(\approx 10 \mathrm{~km})$ heterogeneities has been recently confirmed by Mancinelli and Shearer (2013).

The view that emerges from our understanding of mantle stirring, of plate tectonics, from observations of geochemical heterogeneities and of small scale seismic observations is therefore in agreement with a "marble cake" mantle structure as advocated by Allègre and Turcotte (1986). The mantle should consist of a laminated medium with low velocity contrasts between layers and a power spectrum decreasing as $1 / k$. We want now to compute how much seismic energy could be lost by scattering in such a medium.

\section{Apparent attenuation of a seismic $S$ wave propagating in a laminated structure}

To illustrate the potential effect of small scale heterogeneities on the amplitude of a wave, we consider the simple case of a seismic $\mathrm{S}$ wave propagating perpendicularly along $z$ (polarized in 
the $x y$ plane) through layers of different properties. We will discuss the case of a $\mathrm{P}$ wave, of anisotropy and of non-perpendicular incidences later. The rigidity $\mu(z)$, or density $\rho(z)$ are only functions of $z$. An upgoing wave, incident on $z=0$, with amplitude $\left(S_{u x}(0), S_{u y}(0)\right)^{t}$ is partly reflected by the laminations as a downgoing wave of amplitude $\left(S_{d x}(0), S_{d y}(0)\right)^{t}$ and partly transmitted at the distance $z$ as a wave of amplitude $\left(S_{u x}(z), S_{u y}(z)\right)^{t}$. Transmission $T_{u}$ and reflection $R_{u}$ matrices for the upward propagation can be defined as

$\left(\begin{array}{c}S_{u x}(z) \\ S_{u x}(z)\end{array}\right)=T_{u}\left(\begin{array}{c}S_{u x}(0) \\ S_{u x}(0)\end{array}\right)$

and

$\left(\begin{array}{l}S_{d x}(0) \\ S_{d x}(0)\end{array}\right)=R_{u}\left(\begin{array}{l}S_{u x}(0) \\ S_{u x}(0)\end{array}\right)=R_{u} T_{u}^{-1}\left(\begin{array}{l}S_{u x}(z) \\ S_{u x}(z)\end{array}\right)$.

An incident wave of unit amplitude polarized on the $x$ axis and propagating in such a stack of anisotropic layers will give rise to two reflected waves polarized on both $x$ and $y$ axis, $R_{u x x}$ and $R_{u x y}$, and two transmitted waves, $T_{u x x}$ and $T_{u x y}$. In an isotropic medium, $R_{u}$ and $T_{u}$ are diagonal.

Of course if the structure of the propagating medium were perfectly known, the changes in amplitude of the propagating wave and the existence of a back propagating wave will be correctly interpreted as a purely conservative phenomenon (without dissipation). However if the structure is not accurately known, the change in amplitude of the wave will likely be interpreted as attenuation (scattering attenuation). We will define the equivalent attenuation for a seismic wave with wavenumber $k_{0}$ in our laminated medium as (for the $x$-polarization)

$\frac{S_{u x}\left(k_{0}, z\right)}{S_{u x}\left(k_{0}, 0\right)}=T_{u x x}=\exp \left(-\frac{k_{0} z}{2 Q\left(k_{0}\right)}\right)$.

For each wavenumber $k_{0}$, an attenuation $Q\left(k_{0}\right)^{-1}$ can therefore be computed.

To obtain the reflection and transmission matrices of a complex medium, we use a method related to the "O'Doherty-Anstey" approach (O'Doherty and Anstey, 1971) and discussed in Shapiro et al. (1996). We start from the wave propagation equation transformed in such a way to construct the differential system verified by the transmission and reflection matrices (see Supplementary material A and the differential system (A.12)). This system solved by a standard Runge-Kutta algorithm allows the exact computation of the reflection and transmission properties. Our results have been checked to be identical to those computed by a transfer matrix scheme akin to the Thomson-Haskell method.

The advantage of the O'Doherty-Anstey approach over the Thomson-Haskell method is that we can identify the average propagation (in a homogeneous equivalent isotropic medium with rigidity $\mu_{0}$ and density $\rho_{0}$ ) and the effects of the perturbations due to the variations of rigidity and density along the ray. Assuming that all these perturbations $\delta \mu / \mu$ and $\delta \rho / \rho_{0}$ are small we can derive an analytical estimate of the exact solution by Taylor expansion (see (A.14)). The main result of this cumbersome analytical work is very simple. The equivalent attenuation seen by the wave is simply

$\frac{1}{Q}=\frac{\sqrt{2 \pi}}{4} k_{0} S_{S}\left(2 k_{0}\right)$

where $S_{S}$ is the power spectrum of the quantity.

$\frac{\delta \mu}{\mu}+\frac{\delta \rho}{\rho_{0}}$

(the formalism involves $\delta \rho / \rho_{0}=\left(\rho(z)-\rho_{0}\right) / \rho_{0}$ and $\left(\mu_{0}^{-1}-\right.$ $\left.\left.\mu(z)^{-1}\right) / \mu_{0}^{-1}=\left(\mu(z)-\mu_{0}\right) / \mu(z)=\delta \mu / \mu\right)$. The same approach can be used with P waves (see Supplementary material B). Not surprisingly, we obtain an equivalent attenuation similar to (4) but where the spectrum $S_{S}$ (5) is replaced by the spectrum $S_{P}$ that also involves incompressibility $K$,

$\frac{\delta(K+4 \mu / 3)}{K+4 \mu / 3}+\frac{\delta \rho}{\rho_{0}}$.

This implies that the knowledge of the spectra of heterogeneities in elastic parameters, density and (see later) anisotropy, allows the estimate of the scattering attenuation of the medium.

We assume in this paper that the elastic parameters have small amplitude variations; classically the assumption of effective medium (Backus, 1962; Capdeville and Marigo, 2007) is that the sizes of the heterogeneities are small compared to the wavelength of the seismic wave. The two approaches share however the same mathematical tools (perturbation formalism) and the same physical goals (averaging the perturbations). Notice also that the average equivalent properties of a laminated medium can be obtained numerically by a composite elastic medium theory (Kaelin and Johnson, 1998). It seems however uneasy with this formalism to relate analytically the spectrum of the heterogeneities to the apparent attenuation.

\section{Examples of "elastic" attenuation}

To test the quality of our analytical estimate of the attenuation (4), let us consider the propagation of an elastic wave crossing a 1D medium made of layers of identical thicknesses $h$. In each layer the elastic parameters are uniform, isotropic, but the density is $\rho_{0}+\delta \rho(z)$ where $\delta \rho$ is a small perturbation (this applies to both $\mathrm{S}$ or P waves, see (5) and (6)). We assume that $\delta \rho / \rho_{0}$ is a random variable uniformly distributed over $[-r, r]$. Such a medium is described in Fig. 1(a) where we have chosen $r=\sqrt{3} / 100$ so that the RMS of $\delta \rho / \rho_{0}, \sigma$ is $1 \%$.

The autocorrelation $R(z)$ of such a medium (see definition (A.27)) can be easily found in the limit of an infinite medium

$$
\begin{aligned}
& R(z)=\sigma^{2}\left(1-\frac{|z|}{h}\right) \text { for }|z|<h, \\
& R(z)=0 \text { for }|z|>h .
\end{aligned}
$$

The exact autocorrelation of the function shown in Fig. 1(a) is plotted in Fig. 1(b) with a black line, and its approximation according to (7) with a red line.

The Wiener-Khinchin theorem (A.28) relates the autocorrelation $R(z)$ to the power spectrum $S(k)$ of the medium

$S(k)=\frac{1}{\sqrt{2 \pi}} \int_{-\infty}^{+\infty} R(z) e^{-i k z} d z=\frac{4 \sigma^{2}}{\sqrt{2 \pi}} \frac{\sin ^{2}(k h / 2)}{k^{2} h}$.

The power spectrum of the function depicted in Fig. 1(a) and that given by (8) are shown in Fig. 1(c) (black and red lines).

The expression (4) (in which $S_{S}=S=S_{P}$ ) indicates therefore that the elastic attenuation is of order

$\frac{1}{Q}=\frac{\sqrt{2 \pi}}{4} k_{0} S\left(2 k_{0}\right)=\frac{\sigma^{2}}{4} \frac{\sin ^{2}\left(k_{0} h\right)}{k_{0} h}$.

In Fig. 1(d) we depict this expression as a function of $k_{0}$, (red) and the exact elastic attenuation $-2 \log \left(T_{u x x}\right) /\left(k_{0} z\right)$ (black) obtained by propagating a wave across the $1 \mathrm{D}$ structure of Fig. 1(a). The exact propagation has been computed using the equations (A.12) and with a modified Thomson-Haskell code, the computations give identical results for $\mathrm{S}$ and for $\mathrm{P}$ waves. We can also generate a series of random laminated structures and average the transmission coefficients. This is depicted by the green line of Fig. 1(d) which 

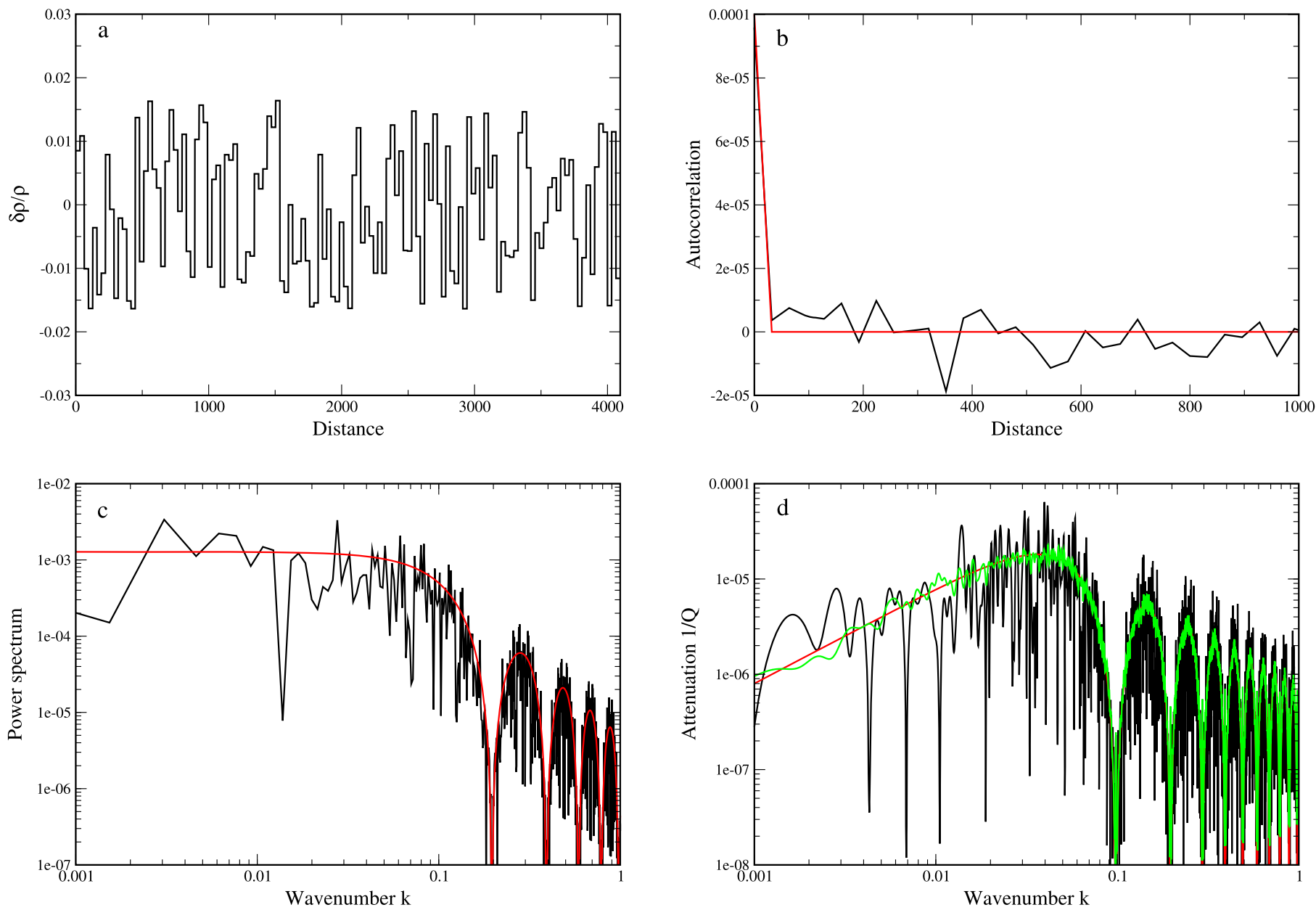

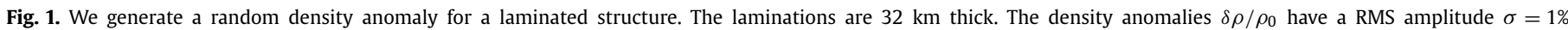

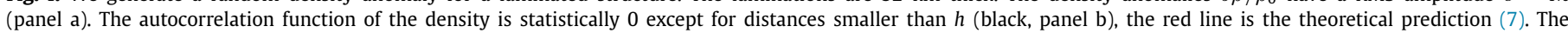

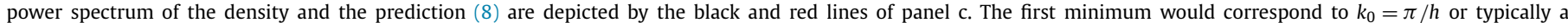

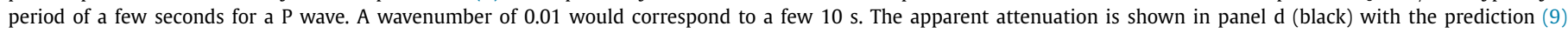

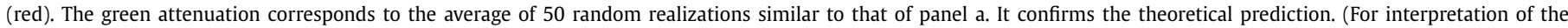
references to color in this figure legend, the reader is referred to the web version of this article.)

averages the attenuations of 50 random distributions (arithmetic average of $Q^{-1}$ ): the statistical distribution of attenuation is identical to the prediction. Although the exact transmission is more complex than that predicted by the approximate solution, it is obvious that we successfully capture the elastic attenuation of the structure.

A more meaningful exercise can be performed for a medium in which the density has a power spectrum in $1 / k$ like what is expected in Earth's mantle. To do so, we first generate Fourier coefficients of the form $\sqrt{S_{0} / k} \exp (i \phi(k))$ where the phase $\phi(k)$ is a random variable uniformly distributed over $\left[0,2 \pi\left[, S_{0}\right.\right.$ a constant and where the wavenumber $k$ is taken between $k_{\min }$ and $k_{\max }$. Then, we perform an inverse Fourier transform of these coefficients. By construction, the power spectrum of the resulting function is $S_{0} / k$ for $k_{\min } \leqslant k \leqslant k_{\max }$; it has a RMS $\sigma$, which according to Parseval's identity is $\sigma^{2}=\sum_{k} S_{0} / k \approx S_{0} \log \left(k_{\max } / k_{\min }\right)$. The elastic attenuation (4) between the wavenumbers $k_{\min } / 2$ and $k_{\max } / 2$ can therefore be written as

$\frac{1}{Q}=\frac{\sqrt{2 \pi}}{8} S_{0}=\frac{\sqrt{2 \pi}}{8} \frac{\sigma^{2}}{\log \left(k_{\max } / k_{\min }\right)}$

where $1 / Q$ is expressed as a function of the amplitude of the heterogeneity spectrum or as a function of the heterogeneity RMS. The function and its spectrum are depicted in Fig. 2, panels (a) and (c), where we have chosen a perturbation RMS of $1 \%$. Considering that Earth's attenuation is constant over 3-4 frequency decades, we choose $k_{\max } / k_{\min }=4096=10^{3.6}$. The autocorrelation function (panel b) is shown in black and the theoretical one (see (A.28)) in red (this function is a cosine integral). In the inserted panel we also use a semilogarithmic scale to show that the autocorrelation is indeed very different from an exponential law (a straight line in semilogarithmic scale). The autocorrelation of heterogeneities in the Batchelor regime decreases much faster than the exponential at short distance but also maintains a significant correlation at long distance. According to (10), the equivalent attenuation (panel (d), black) should be flat (wavenumber or frequency independent). This is the case and the analytical prediction (panel $\mathrm{d}$, red) gives a good fit to the exact attenuation. In green we average 50 random realizations similar to that of panel (a) to confirm the frequency independence of the elastic attenuation. Our analytical estimate seems however to slightly underestimate the average attenuation $\left(4 \times 10^{-6}\right.$ according to $(10)$, instead of $\approx 5 \times 10^{-6}$; compare red and green curves in Fig. 2(d); this may be related to the choice of the averaging, here an arithmetic average of the $Q^{-1}$, geometric or harmonic averages of $Q$ or $Q^{-1}$ give different values also close to the analytical prediction in red). In Fig. 2, there are no units for the horizontal axis and for the power spectrum. There is indeed no characteristic length in this situation and only the ratio $k_{\max } / k_{\min }=4096$ matters. If the distance in panel (a) is in a given unit $(\mathrm{m}, \mathrm{km}, \ldots)$, then the wavenumbers are in unit $^{-1}$, the 

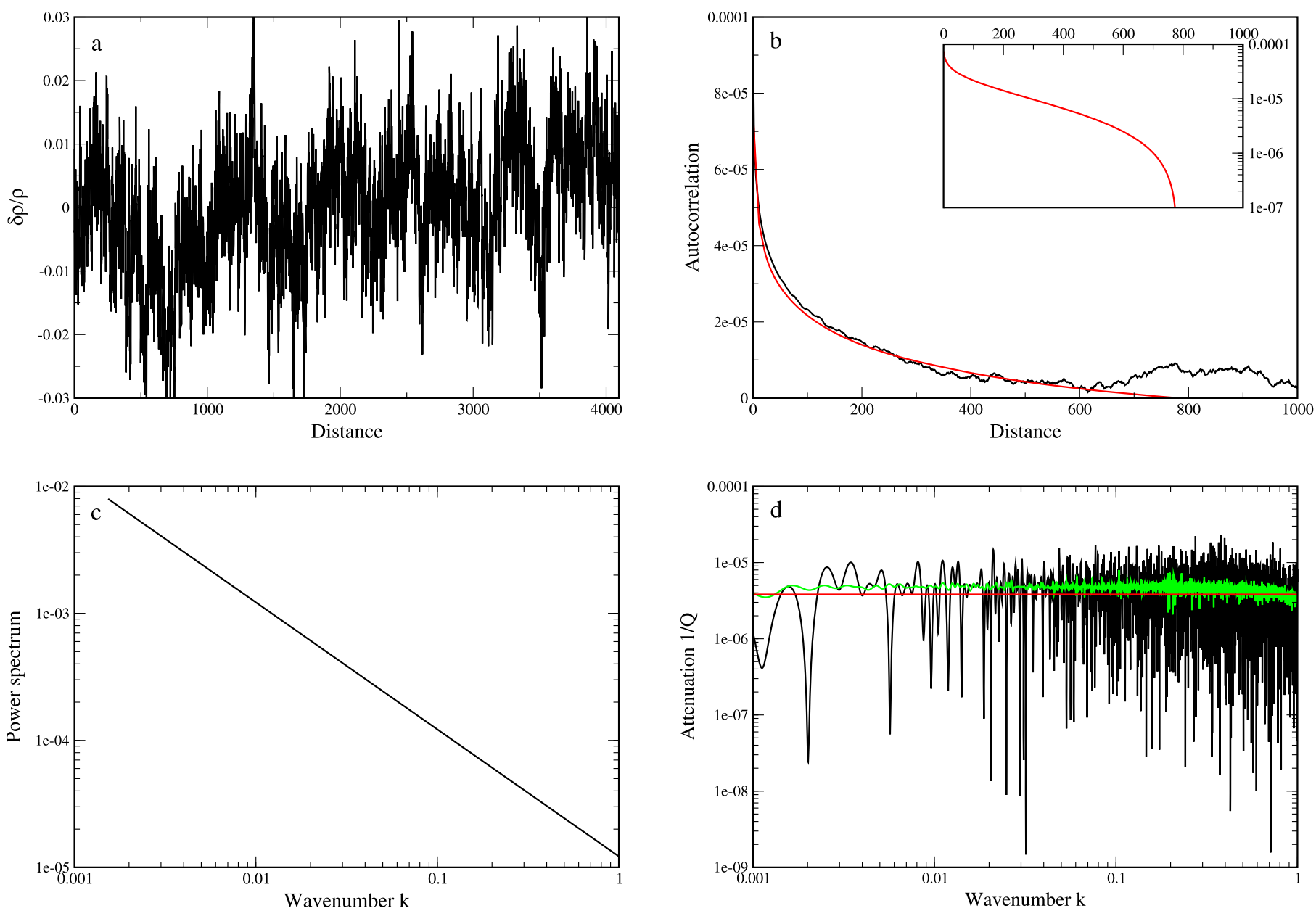

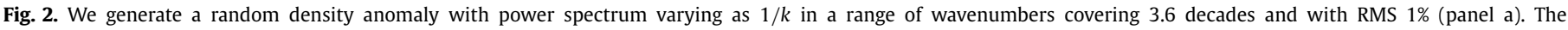

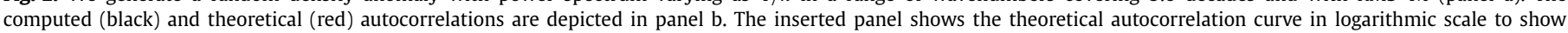

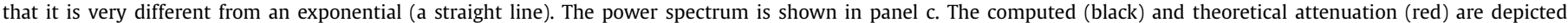

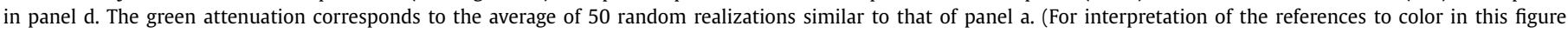
legend, the reader is referred to the web version of this article.)

power spectrum in unit and the same amplitude of attenuation is recovered (but in a wavenumber range defined in unit ${ }^{-1}$ ).

Although we only discussed the elastic attenuation, the propagation of seismic waves in this laminated mantle is also associated with dispersion; the effective propagation velocity is frequency dependent. Our approach implies that attenuation and dispersion are, as they should, related by the usual Kramers-Kronig relations (Waters et al., 2005, see (A.20)). Therefore, if our model is in agreement with the observed attenuation, it is also in agreement with the observed dispersion. For example, assuming a $1 / k$ spectrum of the mantle implies both that the apparent attenuation $1 / Q$ is a constant, and that the phase velocity $v(\omega)$ is frequency dependent with a dispersion deduced from (A.20), $1 / v(\omega)=$ $1 / v_{0}-2 /(\pi Q) \log \left(\omega / \omega_{0}\right)$, which are two assumptions of Prem (Liu et al., 1976). Notice that the heterogeneity spectrum in $1 / k$, which is in agreement with the attenuation and dispersion of Prem, is associated with an autocorrelation function (see Fig. 2(b)) very different from an exponential which has been the hypothesis of several previous studies of mantle scattering.

\section{Attenuation of the mantle}

In the lower mantle $Q_{S}^{-1}$ is found between $1 / 300$ (Prem) and 1/700 (Hwang and Ritsema, 2011; Durand et al., 2013) and $Q_{p}^{-1}$ is of order $4 / 3\left(V_{s} / V_{p}\right)^{2} Q_{s}^{-1}$ i.e., between $1 / 600$ and $1 / 1400$. This last relation results from the observation that the bulk attenuation
$Q_{\kappa}^{-1}$ is very low. In the upper mantle the attenuation is about twice larger than in the lower mantle. It is tempting to compare these values to what can be estimated with our model of elastic attenuation.

In Fig. 2 we obtained $Q^{-1} \approx 5 \times 10^{-6}$ for a $1 \%$ RMS density perturbation, assuming that the medium is isotropic, with constant rigidity and with the same $1 / k$ spectrum over a wavenumber range of 3.6 decades. This range is the typical range of the seismic frequencies over which the observed attenuation seems roughly constant. As this elastic attenuation varies like the amplitude of $\left(\delta \rho / \rho_{0}\right)^{2}$ (see $(10)$ ), we would predict that $17 \%$ to $26 \%$ RMS perturbations of density could explain the observed $S$ attenuation in the lower mantle (12\% to $18 \%$ RMS perturbations for the P attenuation). These RMS values for the density are certainly not reasonable for Earth's mantle anomalies. However, it is not only the density but also the elastic parameters that influence the elastic attenuation.

In mineralogical models (e.g., comparing the properties of basaltic crust and of normal mantle at deep mantle conditions, as in Ricard et al., 2005), the relative contrasts of elastic parameters (assuming isotropy) have similar values that those of density and are generally closely correlated. The power spectra of $\delta \mu / \mu+\delta \rho / \rho_{0}$ or of $\delta(K+4 \mu / 3) /(K+4 \mu / 3)+\delta \rho / \rho_{0}$ are therefore close to 4 times that of density alone (it would be 2 times for uncorrelated variables with similar amplitudes). This would reduce the necessary perturbations needed to explain the whole 
mantle attenuation by elastic attenuation only, by a factor 2 (i.e., 8-13\% density and elastic perturbations to explain the $S$ attenuation, $6-9 \%$ to explain the $\mathrm{P}$ attenuation).

In addition, anisotropy should be considered and in Supplementary material $\mathrm{C}$ we discuss the simple case of transverse anisotropy. Even in a medium where the density and the isotropic velocity are uniform, the presence of anisotropy also induces an elastic attenuation. The shear wave splitting leads to an apparent attenuation estimated from pulse widths or spectra because of the arrival of two quasi-S waves in a window assumed to contain a single $S$ wave, when the period band of measurement is wider than the time separation of the two pulses. This $S$ attenuation is found to be related to the power spectra of $\delta \mu / \mu+\delta \rho / \rho_{0}+\delta a / \mu \cos (2 \psi)$ and of $\delta a / \mu \sin (2 \psi)$ where $a(z)$ is the amplitude of anisotropy (difference between the two rigidities that characterizes the elasticity in this transverse geometry) and $\psi(z)$ the direction of fast polarization in the xoy plane. Assuming that the anisotropy direction is uncorrelated with $\rho$ and $\mu$, the $\mathrm{S}$ attenuation becomes related not only to the power of $\delta \mu / \mu+\delta \rho / \rho_{0}$ but also of $\delta a / \mu$. Taking this effect into account reduces the RMS amplitude of the density and elastic anisotropic parameters necessary to explain both the P and $S$ attenuations by elastic attenuation only, to around 6-9\%.

In Supplementary material D we also consider the case of a non normal incidence $\theta$ to the lamination, in the simple case of a SH wave (so that $\mathrm{S}$ and $\mathrm{P}$ waves remain uncoupled). The elastic attenuation is now a function of the incidence angle, and differs for density variations and for elastic modulus variations. The situation is further complicated because complete reflection can occur when $\theta \rightarrow \pi / 2$. However when density and elasticity heterogeneities are proportional, the final elastic attenuation (D.10) is independent of the incidence angle and therefore identical to the case with normal incidence.

The P-SV case coupling P and S waves is much more cumbersome, but the same method applies as shown in Shapiro et al. (1996). We do not include a supplementary section for this case, as it would be even longer than the 4 supplementary sections already discussed. Invariably we found that the attenuation of both $\mathrm{P}$ and SV waves are now dependent on the combined spectra of density, rigidity and incompressibility, weighted by functions of the incidence angle $\theta$. Although we have not explored all the cases (the transmission and coupling of a $\mathrm{P}$ and general $\mathrm{S}$ wave across a lamination with non-normal incidence), we are confident that for a laminated medium with $1 / k$ spectrum, the elastic attenuation remains frequency independent and with a similar or larger (because the elastic energy can now be exchanged between $\mathrm{P}$ and $\mathrm{S}$ waves) amplitude than with a normal incidence.

The heterogeneities needed to explain the Earth's attenuation by scattering only are large. They are however comparable to what is proposed in the shallow mantle in terms of lateral variations of density (from mineralogy, see Ricard et al., 2005), seismic velocity (e.g., Debayle and Ricard, 2012) or amplitude of anisotropy (e.g., Montagner and Guillot, 2002; Kawakatsu et al., 2011; Debayle and Ricard, 2013), and various localized reflectors with large, positive or negative impedances are observed in the mantle (e.g., Schmandt et al., 2011; Tauzin et al., 2010).

The large RMS heterogeneity that we estimate assumes that the same $1 / k$ heterogeneity spectrum is valid across a wavenumber range of 3.6 decades. It is not directly comparable to the RMS heterogeneity estimates obtained for the lower mantle using high-frequency $(\approx 1 \mathrm{~Hz})$ PKP precursors which only sample a limited number of wavelengths (Margerin and Nolet, 2003; Mancinelli and Shearer, 2013). To compare our model to these PKP precursor studies, we consider like in Mancinelli and Shearer (2013) that the small scale 1D structure has an exponential autocorrelation with a small correlation length $h=6 \mathrm{~km}$. According to the Wiener-Khinchin theorem, (A.28) and the expression of $1 / Q$,
(4), the autocorrelation, the power spectrum and the elastic attenuation are

$$
\begin{aligned}
& R(z)=\sigma^{2} \exp \left(-\frac{z}{h}\right), \\
& S(k)=\frac{2 \sigma^{2}}{\sqrt{2 \pi}} \frac{h}{1+k^{2} h^{2}}, \\
& \frac{1}{Q}=\frac{\sigma^{2}}{2} \frac{k h}{1+4 k^{2} h^{2}} .
\end{aligned}
$$

In Fig. 3, we depict a random function with RMS 1\% and exponential correlation (panels a and b), its power spectrum (d) and the predicted elastic attenuation (d). The result of the numerical simulation is in black, the analytical solution in red, the green lines average 50 random realizations. The maximum of the predicted attenuation corresponds to a wavenumber $k=1 /(2 h)=$ 0.083 (wavelength $4 \pi h \approx 75 \mathrm{~km}$ ) and reaches $\sigma^{2} / 4=1.25 \times 10^{-5}$. Notice that this time, as the heterogeneities are localized in a restricted bandwidth, with the same RMS they lead to a 2.5 larger attenuation than when we assumed that the heterogeneities were distributed over 3.6 decades. Therefore a RMS small scale heterogeneity of $2.4-3.6 \%$ would explain the observed $\mathrm{P}$ attenuation for periods around a few seconds. This is still much larger than what has been suggested for the lower mantle, but would be reasonable for upper mantle heterogeneities.

\section{Conclusion}

The short wavelength content of the mantle heterogeneities is mostly due to petrological anomalies multiply folded by convection and with a power spectrum decreasing as $1 / k$. We present simple models of seismic waves traveling perpendicularly across a 1D laminated structure with this kind of spectrum and show that it results in multiple reflection and in the dispersion of a coherent signal into incoherent noise. The decrease in amplitude of the transmitted wave results in an apparent attenuation (elastic attenuation) that we compute, first, numerically and exactly, and second, using a simple approximated but analytical expression. We show that the elastic attenuation is on average independent of the frequency. This is true whether the density, the elasticity or the anisotropy (keeping uniform isotropic elastic parameters) is the variable varying with a $1 / k$ spectrum. When these quantities vary together in an incoherent fashion, the elastic attenuations due to each variable, sum up. A larger attenuation is obtained when these variables are correlated which is likely the case, at least for density and the isotropic parameters. Similar results should remain valid for a non-normal incidence.

In order to explain the whole attenuation of the mantle by elastic attenuation only and over 3.6 decades of frequency, spatial variations in density and elastic parameters of the order of 6-9 \% are needed. Our model does not discuss the location of these heterogeneities, in the shallow mantle or in the deep Earth. This remains large compared to what is seen in tomography; a few $\%$ in the upper mantle, less than $1 \%$ in the lower mantle, but comparable to the heterogeneity level of the lithosphere. If we reduce the range of frequencies over which we explain the attenuation, we can decrease the amplitude of heterogeneities to levels similar to whose measured in laboratory between different compositions: eclogite/harzburgite have density/elasticity differences in most of the mantle of 2 to 4\% (Irifune and Ringwood, 1993; Ricolleau et al., 2010). Even in this case, the amplitude of these small-scale heterogeneities is much larger that what as been inferred in the deep mantle by previous studies (Margerin and Nolet, 2003; Mancinelli and Shearer, 2013). In the inner core, a level of 

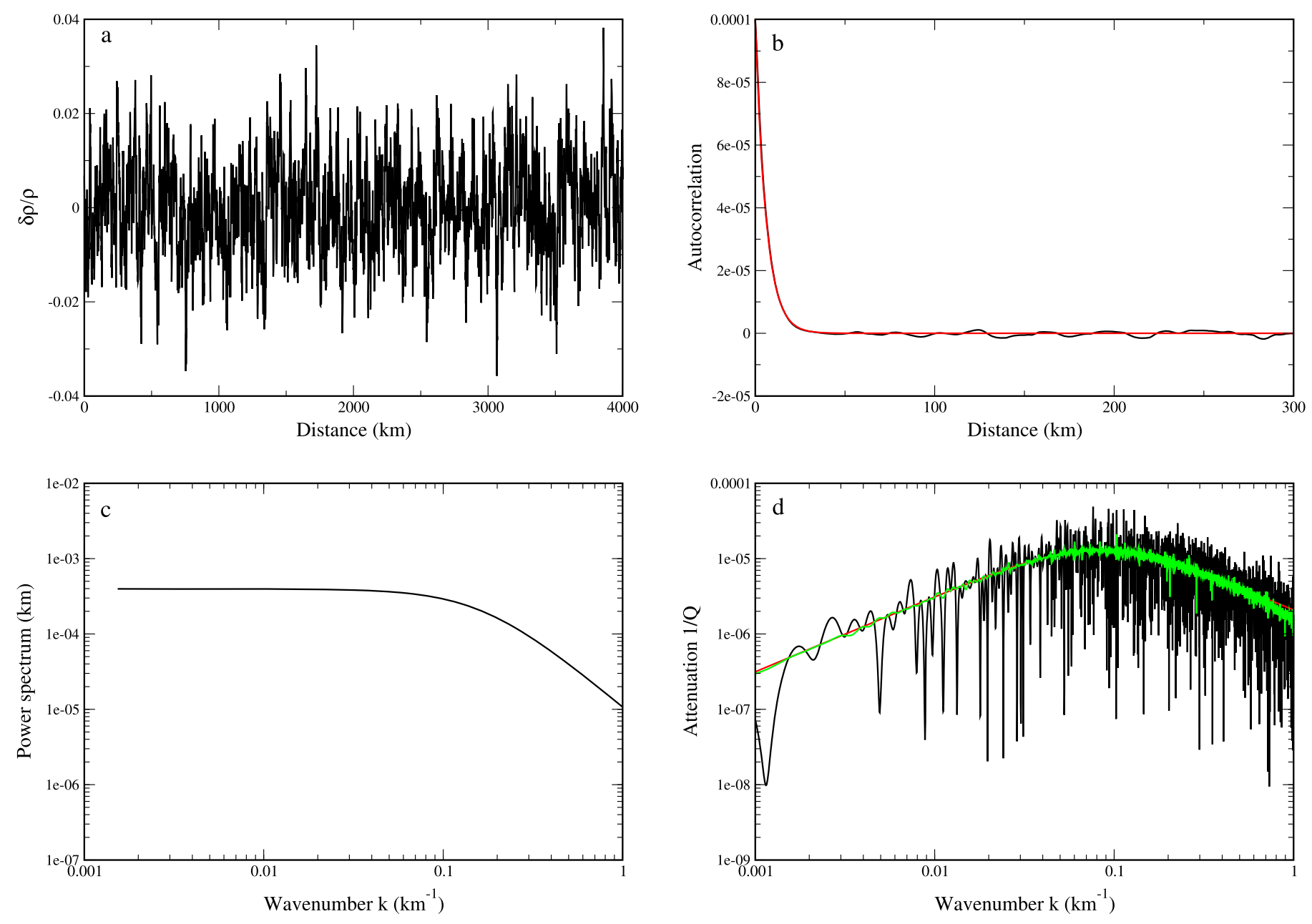

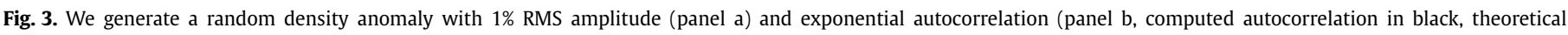

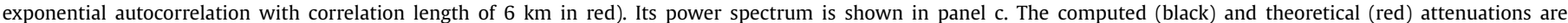

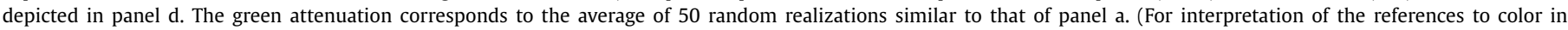
this figure legend, the reader is referred to the web version of this article.)

heterogeneity of a few \% between random patches has been invoked to explain the seismic observations (Cormier and Li, 2002; Calvet and Margerin, 2008).

There are many complexities that we have not taken into account. The P-SV conversions provide another way to distribute the energy incoherently, and would probably increase the apparent attenuation for the same spectrum of heterogeneities. The same would be true when a general anisotropic elastic tensor is considered (while we have only considered transverse anisotropy). It seems that all these complexities will also lead to a similar expression as (4), and a constant attenuation for a medium stirred following Batchelor regime. The fact that the heterogeneities are far from parallel as it has been considered here, should also be taken into account. It seems it should further increase the elastic attenuation.

If most of Earth's attenuation is due to heterogeneities with a $1 / k$ spectrum and a RMS of a few $\%$, then the weak variation of attenuation with frequency would become easy to explain. The fact that $S$ waves are more attenuated than $P$ waves would be simply related to the fact that anisotropy gives $\mathrm{S}$ waves more degrees of freedom to disperse its elastic energy. It would be misleading to interpret these $Q_{P}$ and $Q_{S}$ attenuations in term of $Q_{\kappa}$ and $Q_{\mu}$, as this would wrongly interpret a scattering phenomenon in terms of dissipation. The real dissipative attenuation, that must be present, would be hidden by the elastic attenuation, and the intrin- sic quality factors $Q_{\kappa}$ and $Q_{\mu}$ would simply be higher than what has been observed. Their values might then respect the condition $3 \kappa Q_{\kappa}^{-1}>2 \mu Q_{\mu}^{-1}$ and might not imply a strange auxetic rheology for the mantle.

In principle, the modeling of coda waves could separate the intrinsic and scattering effects (Shearer and Earle, 2004). If the amplitude of heterogeneities necessary to explain the seismic attenuation by elastic scattering implies unrealistically large and complex codas, then it would imply that intrinsic attenuation dominates a frequency independent elastic attenuation. Direct simulation of wave propagation (e.g., within an exact numerical scheme, Komatitsch and Vilotte, 1998) for a 3D structure including small scale heterogeneities, will in a close future be able to model precisely the effect of elastic scattering but computing elastic wave fields up to $\approx 1 \mathrm{~Hz}$ on a global scale will certainly be a challenge.

\section{Acknowledgements}

We thank V. Cormier for his very constructive review and P. Shearer for his meticulous work as editor. This work has been supported by the ANR CMBmelt 10-BLAN-622 and ANR SISMOglob 11-BLAN-SIMI5-6-016-01. J.P. Montagner thanks the Institut Universitaire de France and the training network QUEST. 


\section{Appendix A. Supplementary material}

Supplementary material related to this article can be found online at http://dx.doi.org/10.1016/j.epsl.2013.12.008.

\section{References}

Agranier, A., Blichert-Toft, J., Graham, D., Debaille, V., Schiano, P., Albarède, F., 2005. The spectra of isotopic heterogeneities along the mid-Atlantic Ridge. Earth Planet. Sci. Lett. 238, 96-109.

Allègre, C.J., Turcotte, D.L., 1986. Implications of a two-component marble-cake mantle. Nature 323, 123-127.

Antonsen Jr., T.M., Ott, E., 1991. Multifractal power spectra of passive scalars convected by chaotic fluid flows. Phys. Rev. A 44, 851-857.

Backus, G.E., 1962. Long-wave elastic anisotropy produced by horizontal layering. J. Geophys. Res. 67, 4427-4440.

Batchelor, G.K., 1959. Small-scale variation of convected quantities like temperature in turbulent fluid Part 1. General discussion and the case of small conductivity. J. Fluid Mech. 238, 96-109.

Calvet, M., Margerin, L., 2008. Constraints on grain size and stable iron phases in the uppermost inner core from multiple scattering modeling of seismic velocity and attenuation. Earth Planet. Sci. Lett. 267, 200-212. http://dx.doi.org/10.1016/ j.epsl.2007.11.048.

Capdeville, Y., Marigo, J.J., 2007. Second-order homogenization of the wave equation for non-periodic layered media. Geophys. J. Int. http://dx.doi.org/10.1111/ j.1365-246X.207.03462.x.

Chevrot, S., Montagner, J.P., Snieder, R., 1998. The spectrum of tomographic Earth models. Geophys. J. Int. 133, 783-788.

Choy, G.L., Cormier, V.F., 1986. Direct measurement of the mantle attenuation operator from broad-band P-wave-forms and S-wave-forms. J. Geophys. Res. 91, 7326-7342.

Coltice, N., Ricard, Y., 1999. Geochemical observations and one layer mantle convection. Earth Planet. Sci. Lett. 174, 125-137.

Cormier, V.F., 1995. Time-domain modelling of PKIKP precursors for constraints on the heterogeneity in the lowermost mantle. Geophys. J. Int. 121, 725-736.

Cormier, V.F., Li, X., 2002. Frequency dependent attenuation in the inner core: Part II. A scattering and fabric interpretation. J. Geophys. Res. 107. http://dx.doi.org/ 10.1029/2002JB1796.

Cormier, V.F., 2011. Seismic viscoelastic attenuation. In: Gupta, H. (Ed.), Encyclopedia of Solid Earth Geophysics. Springer, pp. 1279-1290.

Dahlen, F.A., 1982. The effect of data windows on the estimation of free oscillation parameters. Geophys. J. R. Astron. Soc. 69, 537-549.

Debayle, E., Ricard, Y., 2012. A global shear velocity model of the upper mantle from fundamental and higher Rayleigh mode measurements. J. Geophys. Res. 117, B10308. http://dx.doi.org/10.1029/2012JB009288.

Debayle, E., Ricard, Y., 2013. Seismic observations of large-scale deformation at the bottom of fast-moving plates. Earth Planet. Sci. Lett. 376, 165-177. http://dx.doi.org/10.1016/j.epsl.2013.06.025.

Durand, S., Matas, J., Ford, S., Ricard, Y., Romanowicz, B., Montagner, J.P., 2013. Insights from ScS-S measurements on deep mantle attenuation. Earth Planet. Sci. Lett. 374, 101-110. http://dx.doi.org/10.1016/j.epsl.2013.05.026.

Faul, U.H., Jackson, I., 2005. The seismological signature of temperature and grain size variations in the upper mantle. Earth Planet. Sci. Lett. 234, 119-134.

Fichtner, A., Kennett, B.L.N., Trampert, J., 2013. Separating intrinsic and apparent anisotropy. Phys. Earth Planet. Inter. 219, 119-134. http://dx.doi.org/10.1016/ j.pepi.2013.03.006.

Gurnis, M., 1986. Quantitative bounds on the size spectrum of isotopic heterogeneity within the mantle. Nature 323, 317-320.

Hedlin, M.A.H., Shearer, P.M., Earle, P.S., 1997. Seismic evidence for small-scale heterogeneity throughout the Earth's mantle. Nature 387, 145-150.

Hwang, Y.K., Ritsema, J., 2011. Radial $Q_{\mu}$ structure of the lower mantle from teleseismic body-wave spectra. Earth Planet. Sci. Lett. 303, 369-375.

Irifune, T., Ringwood, A., 1993. Phase transformations in subducted oceanic crust and buoyancy relationships at depths of $600-800 \mathrm{~km}$ in the mantle. Earth Planet. Sci. Lett. 117, 101-110.

Jackson, D.D., Anderson, D.L., 1970. Physical mechanisms of seismic-wave attenuation. Rev. Geophys. Space Phys. 8. http://dx.doi.org/10.1029/RG008i001p00001.

Jackson, I., Fitz Gerald, J.D., Faul, U.H., Tan, B.H., 2002. Grain-size-sensitive seismic wave attenuation in polycrystalline olivine. J. Geophys. Res. 107. http://dx.doi. org/10.1029/2001JB001225.

Jackson, I., 2007. Physical Origins of Anelasticity and Attenuation. In: In Schubert, R. (Ed.), Treatise of Geophysics. Elsevier, pp. 493-525.
Kaelin, B., Johnson, L.R., 1998. Dynamic composite elastic medium theory: Part I. One-dimensional media. J. Appl. Phys. 84, 5451-5457. http://dx.doi.org/10.1063/ 1.368307.

Kawakatsu, H., Kumar, P., Takei, Y., Shinohara, M., Kanazawa, T., Araki, E., Suyehiro K., 2011. Seismic evidence for sharp lithosphere-asthenosphere boundaries of oceanic plates. Science 324. http://dx.doi.org/10.1126/science.1169499.

Knopoff, L., 1964. Q. Rev. Geophys. 2, 625-660.

Komatitsch, D., Vilotte, J.P., 1998. The spectral element method: An efficient tool to simulate the seismic response of 2D and 3D geological structures. Bull. Seismol. Soc. Am. 88, 368-392.

Lekic, V., Matas, J., Panning, M., Romanowicz, B., 2009. Measurements and implications of frequency dependence of attenuation. Earth Planet. Sci. Lett. 282, 285-293.

Liu, H.P., Anderson, D.L., Kanamori, H., 1976. Velocity dispersion due to anelasticity: implication for seismology and mantle composition. Geophys. J. R. Astron. Soc. $47,41-58$.

Mancinelli, N.J., Shearer, P.M., 2013. Reconciling discrepancies among estimates of small-scale mantle heterogeneity from PKP precursors. Geophys. J. Int. 195, 1721-1729.

Margerin, L., Nolet, G., 2003. Multiple scattering of high-frequency seismic waves in deep Earth: PKP precursor analysis and inversion for mantle granularity. J. Geophys. Res. 108. http://dx.doi.org/10.1029/2003JB002455.

Montagner, J.-P., 1994. Can seismology tell us anything about convection in the mantle?. Rev. Geophys. 32, 115-137.

Montagner, J.-P., Guillot, L., 2002. Seismic anisotropy and global geodynamics. Rev. Mineral. Geochem. 51, 353-385. http://dx.doi.org/10.2138/gsrmg.51.1.353.

Morozov, I., 2013. http://seisweb.usask.ca/ibm/papers/Q/Morozov_bulk_and_shear_ dissipation.

O’Doherty, R.F., Anstey, N.A., 1971. Reflections on amplitudes. Geophys. Prospect. 19, 430-458.

Olson, P., Yuen, D.A., Balsiger, D., 1984. Mixing of passive heterogeneities by mantle convection. J. Geophys. Res. 89, 425-436.

Ricard, Y., Mattern, E., Matas, J., 2005. Synthetic tomographic images of slabs from mineral physics. In: VanDerHilst, R.D., Bass, J.D., Matas, J., et al. (Eds.), Earth's Deep Mantle: Structure, Composition and Evolution. In: Geophysical Monograph Series, vol. 160, pp. 283-300.

Ricard, Y., Nataf, H.C., Montagner, J.P., 1996. The three-dimensional seismological model a priori constrained: Confrontation with seismic data. J. Geophys. Res. 101, 8457-8472. http://dx.doi.org/10.1029/95JB03744.

Ricolleau, A., Perrillat, J.P., Fiquet, G., Daniel, I., Matas, J., Addad, A., Menguy, N., Cardon, H., Mezouar, M., Guignot, N., 2010. Phase relations and equation of state of a natural MORB: Implications for the density profile of subducted oceanic crust in the Earth's lower mantle. J. Geophys. Res. 115. http://dx.doi.org/ 10.1029/2009JB006709.

Romanowicz, B., Mitchell, B., 2006. Deep Earth structure - Q in the Earth from crust to core. In: Schubert (Ed.), Treatise of Geophysics. Elsevier, pp. 731-774.

Schmandt, B., Dueker, K., Hansen, S., Jasbinsek, J., Zhang, Z., 2011. A sporadic low-velocity layer atop the western U.S. mantle transition zone and shortwavelength variations in transition zone discontinuities. Geochem. Geophys. Geosyst. 12, 1-26.

Shapiro, S.A., Hubral, P., Ursin, B., 1996. Reflectivity/transmissivity for onedimensional inhomogeneous random elastic media: dynamic-equivalentmedium approach. Geophys. J. Int. 126, 194-196.

Shearer, P.M., Earle, P.S., 2008. Observing and modeling elastic scattering in the deep Earth. Adv. Geophys. 50, 167-193. http://dx.doi.org/10.1016/S00652687(08)00006-X.

Shearer, P.M., Earle, P.S., 2004. The global short-period wavefield modelled with a Monte Carlo seismic phonon method. Geophys. J. Int. 158, 1103-1117. http://dx. doi.org/10.1111/j.1365-246X.2004.02378.x.

Shito, A., Karato, S.I., Park, J., 2004. Frequency dependence of Q in Earth's upper mantle inferred from continuous spectra of body wave. Geophys. Res. Lett. 31, L12603. http://dx.doi.org/10.1029/2004GL019582.

Tauzin, B., Debayle, E., Wittlinger, G., 2010. Seismic evidence for a global low velocity layer within the Earth's upper mantle. Nat. Geosci. 3, 718-721.

Wang, N., Montagner, J.P., Fichtner, A., Capdeville, Y., 2013. Intrinsic versus extrinsic seismic anisotropy: The radial anisotropy in reference Earth models. Geophys. Res. Lett. 40, 4284-4288.

Waters, K.R., Mobley, J., Miller, J.G., 2005. Causality-imposed (Kramers-Kronig) relationships between attenuation and dispersion. IEEE Trans. Ultrason. Ferroelectr. Freq. Control 52, 822-833. http://dx.doi.org/10.1109/TUFFC.2005.1503968. 